\title{
BMJ Open Misuse of medication in adult substance misuse services: a systematic review protocol
}

\author{
Rosalind Gittins (D) , ${ }^{1,2}$ Louise Missen, ${ }^{3}$ lan Maidment (D) ${ }^{2}$
}

To cite: Gittins R, Missen L, Maidment I. Misuse of medication in adult substance misuse services: a systematic review protocol. BMJ Open 2021;11:e047283. doi:10.1136/ bmjopen-2020-047283

- Prepublication history and supplemental material for this paper is available online. To view these files, please visit the journal online (http://dx.doi org/10.1136/bmjopen-2020047283).

Received 01 December 2020 Accepted 26 May 2021

Check for updates

(c) Author(s) (or their employer(s)) 2021. Re-use permitted under CC BY-NC. No commercial re-use. See rights and permissions. Published by BMJ.

${ }^{1}$ Clinical Department, Humankind, Durham, UK

${ }^{2}$ Aston Pharmacy School, Aston University, Birmingham, UK

${ }^{3}$ Gilead Sciences, London, UK

Correspondence to

Dr lan Maidment;

i.maidment@aston.ac.uk

\section{ABSTRACT}

Introduction There is a growing concern about the misuse of over the counter (OTC) and prescription only medication (POM) because of the impact on physical and mental health, drug interactions, overdoses and drugrelated deaths. These medicines include opioid analgesics, anxiolytics such as pregabalin and diazepam and antidepressants. This protocol outlines how a systematic review will be undertaken (during June 2021), which aims to examine the literature on the pattern of OTC and POM misuse among adults who are accessing substance misuse treatment services. It will include the types of medication being taken, prevalence and demographic characteristics of people who access treatment services.

Methods and analysis An electronic search will be conducted on the Cochrane, OVID Medline, Pubmed, Scopus and Web of Science databases as well as grey literature. Two independent reviewers will conduct the initial title and abstract screenings, using predetermined criteria for inclusion and exclusion. If selected for inclusion, full-text data extraction will be conducted using a pilottested data extraction form. A third reviewer will resolve disagreements if consensus cannot be reached. Quality and risk of bias assessment will be conducted for all included studies. A qualitative synthesis and summary of the data will be provided. If possible, a meta-analysis with heterogeneity calculation will be conducted; otherwise, Synthesis Without Meta-analysis will be undertaken for quantitative data. The reporting of this protocol follows the Preferred Reporting Items for Systematic Reviews and Meta-Analyses.

Ethics and dissemination Ethical approval is not required. Findings will be peer reviewed, published and shared verbally, electronically and in print, with interested clinicians and policymakers.

PROSPERO registration number CRD42020135216.

\section{INTRODUCTION}

The inappropriate use (misuse) of over the counter (OTC) and prescription only medication $(\mathrm{POM})$, where products are taken (and often the administration route or dose is altered), for nonmedical purposes, is a growing concern internationally. ${ }^{12}$ Although such use of OTC and POMs does not always result in dependency, they may be used in isolation or in combination, including alongside illicit substances and/or alcohol.

\section{Strengths and limitations of this study}

This protocol outlines how a systematic review will be undertaken to provide a comprehensive overview of the patterns of over the counter and prescription only medication misuse by adults who are accessing substance misuse treatment services.

- Two reviewers will independently undertake study selection, data extraction and assessment of risk bias, and a third reviewer will resolve disagreements and review findings.

- The results will include the types of medication being taken, prevalence and associated characteristics, so that the needs of people who access treatment services may be better understood.

- A lack of high-quality studies may be a potential limitation.

- Where available, internationally validated tools will be used for data syntheses and assessing bias risk and quality of the findings.

Concomitant use of medicines, which have additive sedating and respiratory depressant effects, are of particular concern, especially opioids (such as fentanyl and oxycodone), benzodiazepines and gabapentinoids due to the risk of accidental overdose and drugrelated deaths. ${ }^{3-6}$

OTC and POM misuse has also been associated with notable drug interactions, physical and mental health effects, such as psychosis, blood-borne virus transmission, renal failure and QT prolongation (that may result in tachycardias such as torsades de pointes), which may be life threatening. ${ }^{7-9}$ Drug misuse can be associated with significant socioeconomic impact for the person, their friends, family and the wider community. It may lead to incidents that may be significant, such as falls, road traffic collisions and acts of aggression or violence against self or others. ${ }^{10}$

Medication may also be taken to potentiate psychoactive effects or manage side-effects of recreational drug use, such as erectile dysfunction medication for sexual performance associated with methamphetamine. ${ }^{11}$ 
Motivations for OTC and POM misuse may not be solely for recreational or euphoric effect: some people may be self-medicating for withdrawal symptoms, underlying anxiety disorders or pain, as could be the case with baclofen, beta-blockers and opioids, respectively. ${ }^{12-14}$ OTC and POMs may be used to change appearance such as topical steroids to change skin pigmentation ${ }^{15}$ and diuretics, laxatives and stimulants for weight management. ${ }^{16}$ Medicines may also be taken to improve performance, such as cognitive enhancers in academia. ${ }^{17}$

Sometimes the psychoactive effect of OTC and POMs may only become apparent when taken via an alternative route, such as Buscopan (hyoscine N-butyl bromide), which when smoked produces hallucinations, and the antitussive dextromethorphan and loperamide (for diarrhoea) when used at significantly high doses, can have euphoric effects. ${ }^{18-20}$ When being misused, formulations such as buprenorphine tablets may be crushed and administered by injection, ${ }^{21}$ and fentanyl may be extracted from transdermal patches and administered by all routes of administration. ${ }^{22}$ Other examples include codeine, which can be combined with other substances to create new concoctions such as lean ${ }^{23}$ or extracted from combination products using techniques such as 'coldwater extraction'. ${ }^{24}$

In 2016, a report from the UK governments' Advisory Council on the Misuse of Drugs suggested that the misuse of prescription-only painkillers, benzodiazepines, z-drugs and increasingly gabapentinoids is prevalent, with the need for increased vigilance surrounding other medicines including quetiapine, baclofen and stimulants; the need to consider OTC codeine as a 'precursor to the misuse of prescription opioids' was also highlighted. ${ }^{25}$ Misuse may be detected and explored in a variety of ways, using qualitative methodologies such as surveys, interviews, focus groups and netnographic techniques. ${ }^{26-32}$ Qualitative and quantitative information can be obtained from clinical records, surveys, prescribing data, postmarketing surveillance, pharmacovigilance studies and electronic medication dispensing systems. ${ }^{33-36}$ Quantitative data may also be obtained from drug tests, ${ }^{3738}$ and as with any methodology, this has limitations; for example, OTC and POMs may not be detectable or may give false-positive results on drug tests that are routinely used in substance misuse services (SMS). ${ }^{20} 39$

While the misuse of OTC and POMs is thought to be increasing, actual prevalence remains unknown due to a lack of appropriate monitoring systems. ${ }^{25}$ Public Health England (PHE) published the results of their landmark review into dependency and withdrawal associated with some POMs in 2019, which found that that prescribing rates of antidepressants and gabapentinoids among the adult English population in primary care increased in recent years to $15.8 \%$ and $3.3 \%$, respectively: conversely, opioid analgesics, benzodiazepines and z-drugs (such as zopiclone) slightly reduced, though still prevalent with $13 \%, 3 \%$ and $2 \%$, respectively, of the total population currently being prescribed these medicines. ${ }^{40}$
Consequently, the National Institute for Health and Care Excellence is developing guidelines for the management of medicines associated with dependence or withdrawal symptoms. ${ }^{41}$ However, not all medicines that are used inappropriately are necessarily associated with addiction, dependency or withdrawal symptoms, and the PHE review ${ }^{40}$ did not consider all types of medication or different treatment settings such as specialist SMS.

The UK's Clinical Guidelines on Drug Misuse and Dependence ${ }^{42}$ outline that there is a limited evidence base available to inform the clinical management of individuals who require support. This is especially important where illicit substances are used alongside OTC and/or POMs because this may be associated with a lower quality of life and greater mental health issues. ${ }^{43}$ It is, therefore, essential to be able to offer timely and effective interventions to reduce the risk of using other substances, experiencing more severe functional problems and riskier drug-related behaviours. ${ }^{44}$ Additionally, substitute treatment used in SMS may also be used inappropriately and people who access SMS are more likely to experience comorbid mental health conditions, be prescribed psychotropic medication and for unlicensed indications. ${ }^{45} 46$

Data from the USA suggest that once people who need support with their problematic OTC and/or POM use are engaged in treatment, then retention and abstinence rates are thought to be favourable, so identifying their demographic characteristics may assist with improving associated SMS outcomes. ${ }^{47} 48$ The characteristics of people who use OTC and/or POMs may be different in other countries such as the UK and may be different to those who choose to primarily use 'street' drugs; however, people who use 'street' drugs or who may be in receipt of substitute treatments from SMS are also known to misuse OTC and POMs. ${ }^{49} 50$ For example, in the UK, $69 \%$ of people seeking support from SMS are men $^{51}$; however, the 2019 PHE review and the associated publication by Marsden et $a \tilde{l}^{2}$ identified higher rates of prescribing for women of medication associated with dependency and withdrawal. ${ }^{40}$ Other studies have found that women may have a greater propensity for some types of OTC and POM misuse or be more likely to experience more severe outcomes and subsequently account for an increasing proportion of associated emergency department admissions. ${ }^{53-55}$ Furthermore, older age, residing in areas of greater deprivation, comorbid chronic pain issues and HIV-positive status may also increase risk of misuse, but this may vary by the type of medication. ${ }^{40}{ }^{475-59}$ Such medicines may be legally sourced via prescriptions and pharmacy sales and sometimes multiple outlets or healthcare professionals are visited to obtain supplies; however, they may also be obtained illegally, for example, from street dealers or unregulated internet purchases. ${ }^{49}$

There are currently no other known systematic reviews that have focused on the misuse of OTC or POM by people accessing SMS. This protocol is for such a systematic review to be undertaken, in line with Preferred Reporting Items 
for Systematic Reviews and Meta-Analyses (PRISMA), ${ }^{60}$ and using the PRISMA-Protocols (PRISMA-P) checklist. ${ }^{61}$

\section{Aim and objectives}

This protocol outlines how a systematic review will be undertaken, which aims to examine the literature on the pattern of OTC and POM misuse among adults who are accessing substance misuse treatment services. The objectives of the systematic review are to identify the types of medication being taken, the prevalence of use and associated demographic characteristics.

\section{METHODS}

\section{Eligibility criteria}

This review will consist of published studies that must meet all the following criteria:

- Adult participants (18 years or over).

- Individuals receiving interventions for substance misuse in any setting (eg, prison, community, hospital).

- People who are inappropriately using (misusing) OTC and/or POM for nonmedical purposes.

- Individuals in receipt of psychological and/or pharmacological interventions for their substance use.

Prescription medication will not include those that are being prescribed to the individual for the management of their substance misuse disorder. There will be no other restrictions on the type of study population or publication time period. Letters and comments outlining the views, experiences and opinions of individual professionals, researchers, commentators or patients will be excluded; however, reports that summarise and/or collate individual experiences will be considered. For practical reasons, results from all countries and in all languages will be noted; however, only English language results will be considered.

\section{Information sources}

Library staff from Aston University verified the suitability of the search strategy. The reference lists of eligible studies will be manually searched to identify any additional relevant citations to ensure a comprehensive search.

\section{Search}

A comprehensive search of the published literature will be undertaken during June 2021 using the Cochrane, ${ }^{62}$ OVID Medline, ${ }^{63}$ Pubmed,${ }^{64}$ Scopus ${ }^{65}$ and Web of Science Core Collection ${ }^{66}$ databases. Grey literature will also be checked for relevant information. A combination of keywords, medical subject heading and Boolean operator terms related to prescription, and OTC medication misuse in substance misuse treatment will be used such as (Over-the-Counter Drug Misuse) OR (Prescription Drug Misuse) AND (Substance Abuse Treatment Centers). See online supplemental file 1 .

\section{Study selection}

Two independent reviewers (RG and LM) will conduct the initial title and abstract screenings, followed by full-text reviews, using predetermined criteria for inclusion and exclusion. A third reviewer (IM) will resolve disagreements if consensus cannot be reached.

\section{Data collection process}

If selected for inclusion, full-text data extraction will be conducted using a pilot-tested data extraction form. An iterative approach will be taken to improve the usability and to assure the functionality of the forms during the piloting process. Completed pilot forms will be assessed to ensure that they are fit for purpose and that a consistent approach is taken by both reviewers. Where possible, the authors of the papers will be contacted to provide any missing information. The extracted data will be simultaneously captured by both reviewers using Google forms, which all reviewers are familiar with. Patients were not directly involved in the protocol design study and participant recruitment is not required for a systematic review, so disseminating findings to participants is not applicable.

\section{Data items}

The form prompts for retrieval of data including primary author surname, year of publication, title of the publication and journal name, details of medication and other substances, sample size and characteristics, study design, setting, methodology, statistical methods and results, summary of findings and limitations.

\section{Risk of bias}

Quality scores from the Mixed Methods Appraisal Tool (MMAT $)^{67}$ will be used to assess the risk of bias as this tool accommodates quantitative, qualitative and mixed methods, so the same tool can be used for all studies. If appropriate, for systematic reviews, the MMAT will be supplemented by A Measurement Tool to Assess Systematic Reviews version $2 .{ }^{68}$ They will be applied by the reviewers (RG and LM) independently, and the results will be tabulated to enable easy comparison. Similarly, as with data extraction methodology, discrepancies will be discussed and a third reviewer (IM) will be consulted to resolve any disagreements.

\section{Summary measures}

OTC and POM misuse by adults who are receiving treatment for substance misuse may be determined in a multitude of ways. The identification of the types of medication being taken, prevalence and associated demographic characteristics will be considered.

\section{Patient and public involvement}

No patient involved.

\section{Synthesis of results}

All included studies will be appraised with a qualitative summary. The synthesis of qualitative data will follow a thematic analysis approach and the main review findings will be summarised in table format using MS Excel. If the quantitative data allow, a meta-analysis will be undertaken and the outcomes captured in a forest plot. Otherwise, 
numerical descriptors will be captured as a narrative. Quantitative data synthesis methodology and potential for metaanalysis will be dependent on the number and quality of studies identified. Heterogeneity will be assessed using the $\mathrm{I}^{2}$ test, and as outlined by Higgins and Green ${ }^{69}$ heterogeneity is impacted by a variety of factors, so cut-off values will not be enforced; however, heterogeneity will be considered likely if $\mathrm{I}^{2}$ is greater than $40 \%$. If this occurs, subgroup analyses will be undertaken and it may be more likely to occur, for example, for age, gender and type of medication.

As proposed by Campbell et $a l^{70}$, if a meta-analysis is not possible, then Synthesis Without Meta-analysis (SWiM) will be undertaken due to the shortcomings associated with narrative synthesis, and in order to complement PRISMA. Similar to the data extraction process, an independent second review reviewer (LM) will quality assure the analysis, and discrepancies between reviewers will be identified and resolved through discussion (with a third reviewer (IM) where necessary). The resulting cumulative strength of the quality of evidence will be assessed using Grading of Recommendations, Assessment, Development and Evaluation. ${ }^{71}$

\section{Ethics and dissemination}

Ethical approval is not required for a secondary data analysis. The findings will be published in a peer-reviewed journal and at conferences, shared verbally, electronically and in print, with interested clinicians and policymakers.

\section{DISCUSSION}

As all reviewers will be using the same data collection form, which is piloted for suitability of use, this will enable the data extraction process to be standardised. Having two reviewers independently undertaking study selection, data extraction and assessment of risk bias and a third reviewer to resolve disagreements and review findings as well as the use of the $\mathrm{MMAT}^{67}$ will strengthen the quality assurance process.

Internationally validated tools will be used for data syntheses where available. For example, the findings will be reported in line with PRISMA, ${ }^{60}$ and a flow diagram will be used to capture the study selection (and exclusion) process. A lack of high-quality studies may be a limitation, and their diversity may further limit the potential for meta-analyses for quantitative data. If this is the case, then $\mathrm{SWiM}^{70}$ will be used to add robustness.

Undertaking this systematic review will enable a greater understanding of the current published evidence base relating to the pattern of OTC and POM misuse by adults who are accessing SMS. The resulting critique of the findings is important given the increasing concerns about this issue and will be useful to identify areas on which to focus future research.

\section{Twitter Rosalind Gittins @Roz_Gittins and lan Maidment @maidment_dr}

Acknowledgements The authors wish to thank Andrew Doyle, Information Specialist from Aston University Library Services for supporting with development of the search strategy.
Contributors $R G$ conceived the paper and led on writing the initial draft, designed the study, development of data extraction forms, search strategy, critical revision and final review of the manuscript. LM contributed to the manuscript writing, critical revision and final review of the manuscript. IM contributed to the manuscript writing, critical revision and final review of the manuscript. All authors read and approved the final manuscript.

Funding This work presents research funded jointly by the College of Mental Health Pharmacy (CMHP) and Pharmacy Research UK (PRUK-CMHP reference number: PRUK_CMHP-2019-2-RG). The views expressed in this report are those of the authors and not necessarily that of the CMHP or PRUK.

Competing interests None declared.

Patient consent for publication Not required.

Provenance and peer review Not commissioned; externally peer reviewed.

Supplemental material This content has been supplied by the author(s). It has not been vetted by BMJ Publishing Group Limited (BMJ) and may not have been peer-reviewed. Any opinions or recommendations discussed are solely those of the author(s) and are not endorsed by BMJ. BMJ disclaims all liability and responsibility arising from any reliance placed on the content. Where the content includes any translated material, BMJ does not warrant the accuracy and reliability of the translations (including but not limited to local regulations, clinical guidelines, terminology, drug names and drug dosages), and is not responsible for any error and/or omissions arising from translation and adaptation or otherwise.

Open access This is an open access article distributed in accordance with the Creative Commons Attribution Non Commercial (CC BY-NC 4.0) license, which permits others to distribute, remix, adapt, build upon this work non-commercially, and license their derivative works on different terms, provided the original work is properly cited, appropriate credit is given, any changes made indicated, and the use is non-commercial. See: http://creativecommons.org/licenses/by-nc/4.0/.

Author note LM became involved in this work prior to commencing employment at Gilead Sciences, who have no input or involvement in this work.

ORCID iDs

Rosalind Gittins http://orcid.org/0000-0002-0893-7936

lan Maidment http://orcid.org/0000-0003-4152-9704

\section{REFERENCES}

1 Roberts AW, Skinner AC. Assessing the present state and potential of Medicaid controlled substance lock-in programs. J Manag Care Spec Pharm 2014;20:439-46.

2 Winstock A, Strang J. Alternative ways of using and abusing drugs and complicity of doctors. Hosp Med 1999;60:165-8.

3 Jones JD, Mogali S, Comer SD. Polydrug abuse: a review of opioid and benzodiazepine combination use. Drug Alcohol Depend 2012;125:8-18.

4 Lyndon A, Audrey S, Wells C, et al. Risk to heroin users of polydrug use of pregabalin or gabapentin. Addiction 2017;112:1580-9.

5 Macleod J, Steer C, Tilling K, et al. Prescription of benzodiazepines, z-drugs, and gabapentinoids and mortality risk in people receiving opioid agonist treatment: observational study based on the UK clinical practice research Datalink and office for national statistics death records. PLoS Med 2019;16:e1002965.

6 Robinson JA. A crisis hidden in plain sight? prescription opioid misuse in the UK. Pharm J 2017;299 https://pharmaceutical-journal. com/article/feature/a-crisis-hidden-in-plain-sight-prescription-opioidmisuse-in-the-uk

7 Ali M, Mujahid A, Bulathsinghala CP, et al. Cardiac arrhythmia secondary to loperamide abuse and toxicity. Cureus 2020;12:e6936.

8 Amaladoss A, O'Brien S. Cough syrup psychosis. CJEM 2011;13:53-6.

9 Parker BM, Rao T, Matta A, et al. Loperamide induced cardiac arrhythmia successfully supported with veno-arterial ECMO (VA-ECMO), molecular adsorbent recirculating system (MARs) and continuous renal replacement therapy (CRRT). Clin Toxicol 2019;57:1118-22.

10 Modi D, Bhalavat R, Patterson JC. Suicidal and homicidal behaviors related to dextromethorphan abuse in a middle-aged woman. $J$ Addict Med 2013;7:143-4.

11 Hosseinifard SM, Ahmadian A, Smaeelifar N. The synergistic (marathon) effect of combined methamphetamine with sexual stimulant drugs on increasing the likelihood of high-risk sexual behaviors. Addict Health 2014;6:112-8. 
12 Carroll JJ, Rich JD, Green TC. The more things change: Buprenorphine/naloxone diversion continues while treatment remains inaccessible. J Addict Med 2018;12:459-65.

13 Floyd CN, Wood DM, Dargan PI. Baclofen in gamma-hydroxybutyrate withdrawal: patterns of use and online availability. Eur J Clin Pharmacol 2018;74:349-56.

14 Fontanella BJ. Ansiedade social e abuso de propranolol: relato de caso [Social anxiety and propranolol abuse: a case study]. Braz $J$ Psychiatry 2003;25:228-30.

15 Pal D, Biswas P, Das S, et al. Topical steroid Damaged/Dependent face (TSDF): a study from a tertiary care hospital in eastern India. Indian J Dermatol 2018;63:375-9.

16 Austin SB, Penfold RB, Johnson RL, et al. Clinician identification of youth abusing over-the-counter products for weight control in a large U.S. integrated health system. J Eat Disord 2013;1:40. doi:10.1186/2050-2974-1-40

17 McDermott $\mathrm{H}$, Lane $\mathrm{H}$, Alonso M. Working smart: the use of 'cognitive enhancers' by UK university students. J Furth High Educ 2021;45:270-83. doi:10.1080/0309877X.2020.1753179

18 Eggleston W, Marraffa JM, Stork CM, et al. Notes from the field: cardiac dysrhythmias after loperamide abuse - New York, 20082016. MMWR Morb Mortal Wkly Rep 2016;65:1276-7.

19 Jalali F, Afshari R, Babaei A. Smoking crushed hyoscine/scopolamine tablets as drug abuse. Subst Use Misuse 2014;49:793-7.

20 Martinak B, Bolis RA, Black JR, et al. Dextromethorphan in cough syrup: the poor man's psychosis. Psychopharmacol Bull 2017;47:59-63.

21 Varescon I, Vidal-Trécan G, Nabet N. Substitution et mésusage: I'injection intraveineuse de buprénorphine haut dosage [Buprenorphine abuse: high dose intravenous administration of buprenorphine]. Encephale 2002;28:397-402.

22 DrugWatch. Information sheet: fentanyls version: 1.0.1. UK \& Ireland DrugWatch, 2017. Available: http://www.thedrugswheel.com/ drugwatch/Fentanyl_Infosheet_DrugWatch1_0_1.pdf

23 DrugWatch. Information sheet lean (purple Drank/Syrup) version: 1.0 (professionals), 2018. Available: http://thedrugswheel.com/ drugwatch/Lean_Infosheet_DrugWatch_1_0_Pro.pdf

24 Harnett JT, Dines AM, Wood DM, et al. Cold water extraction of codeine/paracetamol combination products: a case series and literature review. Clin Toxicol 2020;58:107-11.

25 Advisory Council on the Misuse of Drugs (ACMD). Diversion and illicit supply of medicines, 2016. Available: https://assets.publishing. service.gov.uk/government/uploads/system/uploads/attachment data/file/580296/Meds report-_final report 15 December LU 2 pdf

26 Cooper RJ. 'I can't be an addict. I am.' Over-the-counter medicine abuse: a qualitative study. BMJ Open 2013;3:e002913.

27 Fingleton NA, Watson MC, Duncan EM, et al. Non-Prescription medicine misuse, abuse and dependence: a cross-sectional survey of the UK general population. J Public Health 2016;38:722-30.

28 Inciardi JA, Surratt HL, Cicero TJ, et al. Prescription opioid abuse and diversion in an urban community: the results of an ultrarapid assessment. Pain Med 2009;10:537-48.

29 Kimergård A, Foley M, Davey Z, et al. Codeine use, dependence and help-seeking behaviour in the UK and ireland: an online crosssectional survey. QJM 2017;110:559-64.

30 Lee E, Cooper RJ. Codeine addiction and Internet forum use and support: qualitative Netnographic study. JMIR Ment Health 2019;6:e12354.

31 Orriols L, Gaillard J, Lapeyre-Mestre M, et al. Evaluation of abuse and dependence on drugs used for self-medication: a pharmacoepidemiological pilot study based on community pharmacies in France. Drug Saf 2009;32:859-73.

32 Van Hout MC, Rich E, Dada S, et al. "Codeine Is My Helper": misuse of and dependence on codeine-containing medicines in South Africa. Qual Health Res 2017;27:341-50. doi:10.1177/1049732315613764

33 Küfner H, Rösner S. Monitoring des Arzneimittelmissbrauchs bei Klienten ambulanter Suchtberatungsstellen (PHAR-MON, ehemals: ebis-med). Monitoring des Arzneimittelmissbrauchs [Monitoring of the misuse of prescription drugs by clients of outpatient addiction treatment centres (PHAR-MON, formerly: Ebis-med). [Monitoring of medication misuse]. Gesundheitswesen 2008;70:305-14.

34 Nielsen S, Roxburgh A, Bruno R, et al. Changes in non-opioid substitution treatment episodes for pharmaceutical opioids and heroin from 2002 to 2011. Drug Alcohol Depend 2015;149:212-9.

35 Wright J, Bond C, Robertson HD, et al. Changes in over-the-counter drug misuse over 20 years: perceptions from Scottish pharmacists. $J$ Public Health 2016:38:793-9.

36 Schifano F, Chiappini S, Corkery JM. Assessing the 2004-2018 fentanyl misusing issues reported to an international range of adverse reporting systems. Front Pharmacol 2019;1:46.
37 McNamara S, Stokes S, Kilduff R, et al. Pregabalin abuse amongst opioid substitution treatment patients. Ir Med J 2015;108:309-10.

38 Peles E, Schreiber S, Linzy S, et al. Differences in methylphenidate abuse rates among methadone maintenance treatment patients in two clinics. J Subst Abuse Treat 2015;54:44-9.

39 Schwartz AR, Pizon AF, Brooks DE. Dextromethorphan-Induced serotonin syndrome. Clin Toxicol 2008;46:771-3.

40 Taylor S, Annand F, Burkinshaw P. Dependence and withdrawal associated with some prescribed medicines: an evidence review. London: Public Health England, 2019. https://assets.publishing. service.gov.uk/government/uploads/system/uploads/attachment data/file/829777/PHE PMR report.pd

41 National Institute for Health and Social Care Excellence (NICE). Medicines associated with dependence or withdrawal symptoms: safe prescribing and withdrawal management for adults, 2019. Available: https://www.nice.org.uk/guidance/gid-ng10141/ documents/final-scope

42 Clinical Guidelines on Drug Misuse and Dependence Update 2017 Independent Expert Working Group (2017). Drug misuse and dependence: UK quidelines on clinical management. London: Department of Health, 2017. https://assets. publishing.service.gov.uk/ government/uploads/system/uploads/attachment_data/file/673978/ clinical guidelines 2017.pdf

43 Pv A, Abdin E, Jun Wen T, et al. Correlates of non-medical prescription drug misuse among a treatment-seeking population: a comparison with illicit drug users. Int J Environ Res Public Health 2018;15:1978.

44 Butler SF, Black RA, Serrano JMG, et al. Characteristics of prescription opioid abusers in treatment: prescription opioid use history, age, use patterns, and functional severity. J Opioid Manag 2010;6:239-41, 246-52.

45 Gale-Grant O, Bailey J, Burke O, et al. Use of prescribed psychotropic medications in an opioid substitution therapy cohort. $J$ Dual Diagn 2019;15:254-9.

46 Reimer J, Wright N, Somaini L, et al. The impact of misuse and diversion of opioid substitution treatment medicines: evidence review and expert consensus. Eur Addict Res 2016;22:99-106.

47 McCabe BE, Santisteban DA, Mena MP, et al. Engagement, retention, and abstinence for three types of opioid users in Florida. Subst Use Misuse 2013;48:623-34.

48 Rigg KK, Monnat SM. Comparing characteristics of prescription painkiller misusers and heroin users in the United States. Addict Behav 2015;51:106-12.

49 Rosenblum A, Parrino M, Schnoll SH, et al. Prescription opioid abuse among enrollees into methadone maintenance treatment. Drug Alcohol Depend 2007;90:64-71.

50 Tkacz J, Ruetsch C. PMH59 differences in baseline problem severity between prescription and street opioid abusers ameliorated after participation in disease management program: results at one year. Value in Health 2011;14:A196.

51 Public Health England (PHE). Adult substance misuse treatment statistics 2018 to 2019: report, 2019. Available: https://www.gov. uk/government/publications/substance-misuse-treatment-foradults-statistics-2018-to-2019/adult-substance-misuse-treatmentstatistics-2018-to-2019-report\#people-in-treatment-substance-sexage

52 Marsden J, White M, Annand F, et al. Medicines associated with dependence or withdrawal: a mixed-methods public health review and national database study in England. Lancet Psychiatry 2019:6:935-50.

53 Carey J, Boyle KL, Rhyee S. TX 758 gender differences in prescription medication abuse. SAEM annual meeting 2014Dallas, 2014. Available: https://www.acmt.net/_Library/ToxIC/ FINALsaemabstract_for_ToxIC.pdf

54 Scholz I, Schmid Y, Exadaktylos AK, et al. Emergency department presentations related to abuse of prescription and over-the-counter drugs in Switzerland: time trends, sex and age distribution. Swiss Med Wkly 2019;149:w20056.

55 Simoni-Wastila $\mathrm{L}$. The use of abusable prescription drugs: the role of gender. J Womens Health Gend Based Med 2000;9:289-97.

56 Chhatre S, Cook R, Mallik E, et al. Trends in substance use admissions among older adults. BMC Health Serv Res 2017; $17: 584$

57 Dhokia M, Elander J, Clements K, et al. A randomized-controlled pilot trial of an online compassionate mind training intervention to help people with chronic pain avoid analgesic misuse. Psychol Addict Behav 2020;34:726-33.

58 Passik SD, Schreiber J, Kirsh KL, et al. A chart review of the ordering and documentation of urine toxicology screens in a cancer center: do they influence patient management? J Pain Symptom Manage 2000;19:40-4. 
59 Satre DD. Alcohol and drug use problems among older adults. Clinical Psychology: Science and Practice 2015;22:238-54.

60 Moher D, Liberati A, Tetzlaff J, et al. Preferred reporting items for systematic reviews and meta-analyses: the PRISMA statement. PLoS Med 2009;6:e1000097.

61 Moher D, Shamseer L, Clarke M, et al. Preferred reporting items for systematic review and meta-analysis protocols (PRISMA-P) 2015 statement. Syst Rev 2015;4:1.

62 Wiley. Cochrane library advanced search, 2021. Available: https:// www.cochranelibrary.com/advanced-search

63 Wolters Kluwer. Ovid search home page. available from, 2021. Available: https://ovidsp.ovid.com/ (Accessed 18/02/21)

64 National Library of Medicine. Pubmed search home page, 2021. Available: https://pubmed.ncbi.nlm.nih.gov/

65 Elsevier. Scopus preview home page, 2020. Available: https://www. scopus.com/standard/marketing.uri

66 Clarivate Analytics. Web of science service, 2020. Available: https:// wok.mimas.ac.uk/
67 et alHong QN, Pluye P, Bregues SF. Mixed methods appraisal tool (MMAT) user guide. McGill department of family medicine, 2018. Available: http://mixedmethodsappraisaltoolpublic.pbworks.com/w/file/ fetch/127916259/MMAT 2018 criteria-manual 2018-08-01_ENG.pdf

68 Shea BJ, Reeves BC, Wells G, et al. AMSTAR 2: a critical appraisal tool for systematic reviews that include randomised or nonrandomised studies of healthcare interventions, or both. BMJ 2017;358:j4008.

69 Higgins JPT, Green S. Cochrane Handbook for systematic reviews of interventions version 5.1.0. London: The Cochrane Collaboration, 2011. http://handbook.cochrane.org

70 Campbell M, McKenzie JE, Sowden A, et al. Synthesis without meta-analysis (swim) in systematic reviews: reporting guideline. BMJ 2020;368:16890.

71 Balshem H, Helfand M, Schünemann HJ, et al. Grade guidelines: 3 rating the quality of evidence. J Clin Epidemiol 2011;64:401-6. 